\section{L'epidemia ai nostri tempi}

\author{
Marco Lombardi \\ Editor in Chief, Giornale di Clinica Nefrologica e Dialisi
}

Questo maledetto virus, oltre a non averci ancora lasciato, è anzi ritornato più potente e pressante di prima. Oltre a non averci ancora lasciato, sta producendo un cambiamento interno in ognuno di noi, non sempre palese ma che temo si manifesterà sempre più nel prossimo futuro.

Lo vedo e lo sento nei rapporti con i colleghi, ma anche nei soggetti che devo assistere e curare; lo sento dentro di me sto cambiando - me ne accorgo con i famigliari (i parenti e gli amici sono spesso 'tenuti' alla lontana per precauzione); non si parla - non si pensa - ad altro!

Se il maledetto incide negativamente anche sulla narrativa (ad esempio, il concorso è rimandato al 2021 ed ipotizzato in forma diversa) è proprio della narrativa che c'è più bisogno.

La narrativa ha da sempre forme diverse per essere attuata. In questo momento, grazie anche all'allenamento a fare visite nell'ambulatorio di predialisi e di nefrologia narrativa con gli amici e colleghi Alessandro Toccafondi (Psicologo clinico) e Leonardo Mari (Farmacista ospedaliero, Counsellor e, non ultimo, poeta), sento la necessità di essere più narrativo o meno medico tradizionale e paternalista possibile (quando ci riesco... certo non sempre!).

È veramente palpabile il bisogno di tutti di essere capiti e di capire, ma soprattutto di essere ascoltati.

Se nel mio piccolo e nella mia piccola pratica medica la narrativa diviene un'esigenza anche per combattere le sequele Covid-19, la poesia può essere, anzi è, narrativa allo stato puro e pertanto ottimo rimedio per esprimere, manifestare, risvegliare, farsi ascoltare.

Ho chiesto a due poeti a me vicini per motivi diversi, ma sempre legati alla mia 'specifica' di scrivere qualcosa per la rubrica di Nefrologia Narrativa del Giornale, qualcosa che ovviamente rappresentasse il loro stato d'animo e lo stato del sentire generale in questo momento così particolare.

Grazie Ivo, grazie Leo!

Received: November 18, 2020

Accepted: November 18, 2020

Published online: December 7, 2020

Indirizzo per la corrispondenza:

Marco Lombardi

Ospedale del Mugello

Via Della Resistenza, 60

50032 Borgo San Lorenzo, Firenze - Italia

lombardim@tin.it

\section{Inquietudine delle ombre}

\section{Leonardo Mari}

Farmacia dell'Ospedale del Mugello, Azienda USL Toscana Centro, Firenze - Italia
Il corto rumore del tempo
e le mani, le mani
a coprire la faccia
per la vergogna d'esistere
per l'impotenza di assistere
inerme alla scure del boia.
C'è una Croce sbiadita
dalla legge dell'uomo
che respira a fatica
in questo pantano
mentre ai calici bolle
un nettare scuro
che avvelena le labbra
di un potere infernale.
Nient'altro ho da dire e forse troppo ho già detto perché questa colpa la sento anche mia: son pronto a tradire cosciente e incosciente con gli occhi e col cuore dell'uomo di sempre.

\section{Pensieri del Poeta al ritorno della tempesta}

\section{Ivo Guasti}

Poeta in Barberino del Mugello, Firenze - Italia

Hanno cuori che vivono le amiche case del tempo sbrecciati muri dai suoni del vento e il lungo fiato alle porte rassicura l'andare e il ritorno degli uomini.
Il treno fischia nell'animo avanza nella nebbia fugge notti portando giorni come sempre la vita lenta o veloce così inesorabile.
Sembriamo alberi nel vento immobili a sfidare la tempesta salvati dalla volontà d'esistere. 